\title{
ENERGY EFFICIENT ROUTING USED FOR WIRELESS SENSOR NETWORKS EXPLOITATION IN GENETIC ALGORITHMIC PROGRAM
}

\author{
K.Murugesan ${ }^{1}$, S.Jagatheswaran ${ }^{2}$, P.Kanagaraj ${ }^{3}$, G.Renuka ${ }^{4}$ \\ ${ }^{1} P G$ Student, K.S.R College of Engineering, Tamilnadu, India \\ ${ }^{2} P G$ Student, K.S.R College of Engineering, Tamilnadu, India \\ ${ }^{3} P G$ Student, K.S.R College of Engineering, Tamilnadu, India \\ ${ }^{4} P G$ Student, K.S.R College of Engineering, Tamilnadu, India
}

\begin{abstract}
Life time of Wireless device Networks (WSNs) has perpetually been a important issue and has received enlarged attention within the recent years. Typically wireless device nodes area unit equipped with low power batteries that area unit impossible to recharge. Wireless device networks ought to have enough energy to satisfy the specified necessities of applications. during this paper, we have a tendency to propose Energy economical Routing and Fault node Replacement (EERFNR) formula to extend the lifespan of wireless device network, cut back information loss and conjointly cut back device node replacement value. Transmission drawback and device node loading drawback is solved by adding many relay nodes and composition device node's routing mistreatment stratified Gradient Diffusion. The device node will save backup nodes to cut back the energy for re-looking the route once the device node routing is broken. Genetic formula can calculate the device nodes to exchange, apply the foremost on the market routing methods to replace the fewest device nodes.
\end{abstract}

Keywords: Genetic algorithmic rule, stratified gradient diffusion, grade diffusion, wireless device networks ****

\section{INTRODUCTION}

Wireless device Network (WSN) may be a cluster of wireless device nodes that have tiny capacities of sensing, process that ar deployed over a region for sensing natural phenomenon. Usually, these device nodes send their perceived information to a base station for any process. They are prepared with low value tiny capability batteries that square measure, nonrechargeable and irreplaceable. Hence, network period of time is taken into account as a crucial issue for several applications. many routing algorithms for the wireless device network are consecutive planned in recent years. C. Intanagonwiwat et al. given the Directed Diffusion (DD) algorithmic program [2] in 2003. The Doctor of Divinity algorithmic program aims to scale back transmission counts of knowledge relay and energy consumption. Basically, the Doctor of Divinity algorithmic program may be a question driven transmission protocol within which the collected knowledge is transmitted to sink node given that the collected knowledge is matched with the question from the destination node, thus the ability consumption of the transmission is reduced. In 2011, H. C. Shih et al. [10] planned a ladder diffusion algorithmic program victimization hymenopterous insect colony optimisation for wireless device networks (LD-ACO) to unravel the routing and energy consumption downside. Moreover, the LD-ACO algorithmic program will improve the device node's period of time
.The LD algorithmic program produces the ladder table in every device node supported the whole wireless device network by issuance the ladder in create packet that's created from the sink node. once the ladder diffusion method, they planned AN improved hymenopterous insect colony optimisation algorithmic program to equalization the information transmission load, increasing the period of time of device nodes. Shengxiang rule et al. planned Genetic algorithmic program with immigrants and memory theme to unravel dynamic routing downside for mobile ad hoc networks. This immigrants and memory-based GAs will quickly adapt to environmental changes (i.e., the constellation changes) and manufacture high-quality solutions once every change[5]. once the random deployment of sensors within the target, the matter of finding the most important variety of disjoint sets of sensors, with each set being in a position to fully cowl the target, is nondeterministic polynomialcomplete. Xiao-Min Hu et al. projected a hybrid approach of mixing a genetic algorithmic program with schedule transition operations (STHGA) to unravel this downside and construct energy economical wireless device networks [4]. Hong-Chi Shih et al. projected a fault node recovery algorithmic program to boost the period of a wireless device network once a number of the device nodes pack up. The algorithmic program relies on the grade diffusion algorithmic program combined with the genetic algorithm [2]. Within the wireless device network (WSN), reduction of energy consumption is extremely necessary for every device node as a result of it will 
extend WSN period. If some device nodes can't work in the WSN, the routing path can break and also the detected space can have leaks. Moreover, different device nodes can't transfer event information to the sink node, or they have a lot of device nodes to present them help. device nodes close to the sink node area unit known as -inside nodell et al. area unit known as -outside nodell. we are able to realize that the surface nodes of WSN would like within nodes to present them help once outside nodes transfer information to the sink node. Hence, the within nodes have vast loading, and their energy are consumed quickly. once the within nodes area unit out of energy, there's no device node which will transfer information to the sink node, and also the WSN are out of operate. during this paper, we tend to projected a classconscious gradient diffusion (HGD) algorithmic program with genetic algorithmic program (GA) to enhance the whole WSN period.

\section{ENERGY ECONOMICAL ROUTING AND} FAULT NODE REPLACEMENT ALGORITHMIC

\section{PROGRAM}

This paper proposes Energy economical Routing and Fault Node Replacement rule (EERFNR) rule for WSNs supported the gradable gradient diffusion rule combined with the genetic rule. The flowchart is shown in Fig. 1. The EERFNR rule creates the grade worth, routing table, neighbor nodes, and payload worth for every device node exploitation the gradable gradient diffusion rule. Fig. 1, the EERFNR rule creates the grade worth, routing table, a collection of neighbor nodes, and payload worth for every device node, exploitation the hierarchal gradient diffusion rule. The Device nodes transfer the event knowledge to the sink node consistent with the GD rule once events seem. If the variety of non functioning nodes exceeds the brink price then genetic rule is invoked to interchange the nonfunctional nodes by useful nodes and employ the foremost on the market path.

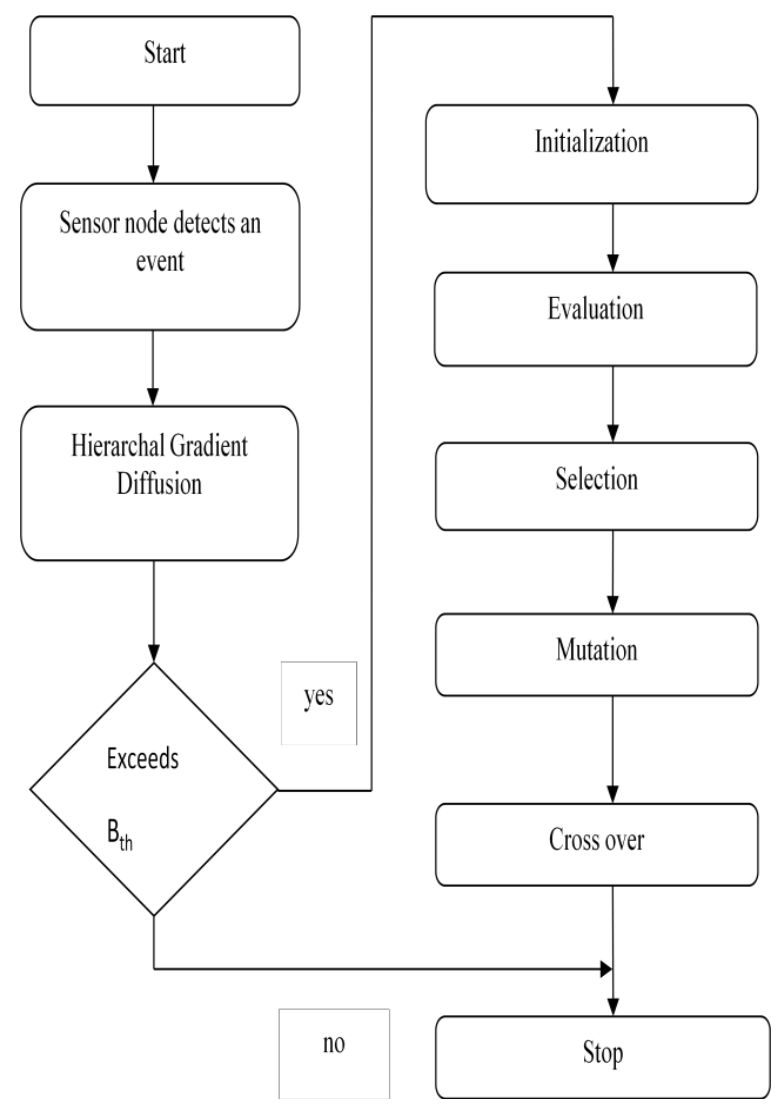

Fig1. Steps in Energy proficient Routing

\section{HIERARCHICAL GRADIENT DIFFUSION ALGORITHM}

The HGD formula adds some RS nodes that square measure relay nodes of the sink node and that they will broadcast the grade making package because the sink node. device nodes will transfer knowledge to RS nodes or the sink node to balance device node loading, reducing the energy consumption and enhancing WSN period according to the HGD formula. Moreover, device nodes will save backup nodes in its routing table to scale back the energy for the relooking routing by our planned formula just in case the device node's routing is broken. The RS node is comparable to the sink node as a result of it doesn't have any detection ability; they will simply be a knowledge assortment center for device nodes in addition because the sink node. Moreover, the RS nodes have giant transmission scale compared with device nodes, and that they have enough energy to transfer knowledge to real knowledge assortment center (Sink Node). Hence, events may be detected and transferred to RS nodes or the sink node by device node. If associate degree RS node receives a happening knowledge, the event knowledge are transferred to the sink node from RS node. Hence, device node, RS node, and sink node become a hierarchical data structure within the HGD formula. In HGD formula, the grade making package are broadcasted from the sink node RS nodes. 
Firstly, the sink node broadcasts grade- making packages to make a main routing table for device nodes. Then, RS node broadcasts grade- making packages once more to make a backup routing table. Moreover, device nodes will amendment their main routing table and backup routing table per the grade data received from grade-creating packages. Thus, the routing path may be hamper and therefore the transmission loading may be reduced once the routing path from device node to RS node is shorter than to sink node. Firstly, the sink node broadcasts the grade-creating package and therefore the package format. within the grade-creating package format, as shown in figure two, the SRS mean sink node holds the worth is 0 , otherwise it's a grade price of RS node.

\begin{tabular}{|l|l|l|}
\hline SRS & HCP & DN \\
\hline
\end{tabular}

Fig 2 Grade Creating Packaging

The HCP suggests that what percentage hop counts a device will transfer event knowledge to the sink node or RS node. The DN suggests that the destination node, and therefore the destination is that the sink node or RS node.

\section{GENETICAL ALGORITHM}

The parameters ar encoded in binary string and function the chromosomes for the GA. the weather (or bits),i.e., the genes, within the binary strings ar adjusted to reduce or maximize the fitness worth. The fitness perform generates its fitness worth, that consists of multiple variables to be optimized by the GA. At every iteration of the GA, a planned range of people can manufacture fitness values related to the chromosomes.

The following may be a typical GA procedure: Procedure GA Begin

Initialize population;

Evaluate population members;

While termination condition not happy do

Begin

Select oldsters from current population;

Apply genetic operators to choose parents;

Appraise offspring;

Set offspring adequate current population;

Finish

End

There are five steps in the genetic rule as represented below.

\subsection{Data Format}

In the data format step, the genetic rule generates chromosomes, and everybody is associate degree expected answer. the amount of chromosomes is set in line with the population size, that is outlined by the user. Everybody is a combination answer, and also the body length is that the range of sensing element nodes that ar depleted or nonfunctioning the weather within the genes ar either 0 or 1 . A one suggests that the node ought to get replaced, and means the node won't get replaced.

\subsection{Evaluation}

In general, the fitness worth is calculated per a fitness operate, and additionally the parameters of the fitness operate ar the chromosome's genes. However, we tend to tend to cannot place genes directly into the fitness operate among the EERFNR formula, as a results of the genes of the body ar just whether or not or not the node got to get replaced or not. among the EERFNR formula, the goal is in addition to recycle the foremost routing strategies and to interchange the fewest device nodes. Hence, the variability of routing strategies out there if some nonfunctioning device nodes ar replaced is calculated, and additionally the fitness operate is shown as below.

$$
\mathrm{F}(\mathrm{n})=\sum_{i=1}^{\max g r a d e} \frac{R P i \times T S N}{S N i \times T R P \times i}
$$

Where

$\mathrm{SNi}=$ the quantity of replaced detector nodes and their grade worth at $\mathrm{i}$.

$\mathrm{RPi}=$ the quantity of re-usable routing ways from detector nodes with their grade worth at $i$.

TSN $=$ total range of detector nodes within the original WSN. $\mathrm{TRP}=$ total range of routing ways within the original WSN. A high fitness worth is wanted as a result of the WSN is yearning for the foremost obtainable routing ways and therefore the least range of replaced detector nodes.

\subsection{Selection}

The selection step can eliminate the chromosomes with very cheap fitness values and retain the remainder. we tend to use the political orientation strategy and keep the $1 / 2$ the chromosomes with higher fitness values and place them within the coupling pool. the more serious chromosomes are deleted, and new chromosomes will be made to replace them after the cross overstep.

\subsection{Crossover}

The crossover step is employed within the genetic algorithmic program to vary the individual body. during this algorithmic program, we have a tendency to use the one-point crossover strategy to make new chromosomes. 2 individual chromosomes square measure chosen from the pairing pool to supply 2 new offspring. A crossover purpose is chosen between the primary and last genes of the parent people. Then, the fraction of every individual on either facet of the crossover purpose is changed and concatenated. the speed of selection is 
formed in keeping with roulette-wheel choice and therefore the fitness values.

\subsection{Mutation}

The mutation step will introduce traits not found within the original people and prevents the GA from converging too quickly. During this rule, we have a tendency to merely flip a factor at random within the body. The body with the best fitness price is the answer once the iteration. The EERFNR rule can replace the detector nodes within the body with genes of one to increase the WSN lifespan.

\section{CONCLUSIONS}

In this paper, Energy economical Routing and Fault Node Replacement (EERFNR) algorithmic program is projected for wireless detector network to extend the life time, cut back knowledge loss and node cost. Grade worth, routing table, neighbor nodes, payload worth for every node is made by hierarchic gradient diffusion and it conjointly add some relay nodes \{to cut back|to scale back|to cut back\} the load of internal nodes and reduce knowledge loss owing to large load of internal nodes. Then non functioning detector nodes ar replaced by functioning detector nodes and most obtainable routing methods ar used by genetic algorithmic program to cut back the node cost and knowledge loss.

\section{REFERENCES}

[1]. Hong-ChiShih,Jiun-HueiHo, Bin-YihLiao, and JengShyangPan-Fault node recovery algorithm for wireless sensor networking \|IEEE sensors journal, vol.13,no.7,july2013 [2]. Chalermek Intanagonwiwat, Ramesh Govindan, Deborah Estrin, John Heidemann, and Fabio Silvall Directed Diffusion for Wireless Sensor Networking\| IEEE/ACM Transactions on Networking11, 2-16 (2003).

[3]. Hong-ChiShih,Jiun-HueiHo, Bin-YihLiao, and JengShyang Pan\|-Hierarchical Gradient Diffusion Algorithm for Wireless Sensor Networks-SpringerVerlagBerlinHeidelberg2013.

[4]. Xiao-Min $\mathrm{Hu}$, Jun Zhang, Yan Yu $\|$ Hybrid GeneticAlgorithm UsingaForwardEncoding Scheme for Lifetime Maximization of Wireless Sensor Networks\| IEEE Transactions On Evolutionary Computation,Vol.14,No.5,October 2010.

[5]. Shengxiang Yang,HuiCheng, and Fang Wang\| Genetic Algorithms With Immigrants and Memory Schemes for Dynamic Shortest Path Routing Problems in Mobile AdHoc Networks\| IEEE Transactions On Systems, Man, And Cybernetics - part C: Applications AndReviews,Vol.40,No.1, January2010.

[6]. YinWu,WenboLiu-Routing protocol based on Genetic algorithm for Energy Harvesting -Wireless sensornetworks\|IETWirel.Sens.Syst.,2013,Vol.3, Iss.2, pp.112-118.
[7]. Tian-huaLiu,Si-chaoYi-AFault Management Protocol for Low-Energy and Efficient Wireless Sensor Networks\|Journal of Information Hiding and Multimedia Signal Processing Volume4,Number 1, January2013.

[8]. M.Ashouri,Z.Zali, S.R.Mousavi-New optimal solution to disjoint set k coverage for lifetime extension in wireless sensor networks\| IET Wirel. Sens.Syst., 2012, Vol.2.

[9]. J.H.Ho,H.C.Shih,B.Y.Liao, andS.C.Chu,-Aladder diffusion algorithm using ant colony optimization for wireless sensor networks, ॥Inf. Sci., vol.192,pp.204-212,Jun.2012. 